\title{
Anyon Hubbard Model in One-Dimensional Optical Lattices
}

\author{
Sebastian Greschner and Luis Santos \\ Institut für Theoretische Physik, Leibniz. Universität Hannover, Appelstraße 2, DE-30167 Hannover, Germany
}

(Received 5 February 2015; published 28 July 2015)

\begin{abstract}
Raman-assisted hopping may be used to realize the anyon Hubbard model in one-dimensional optical lattices. We propose a feasible scenario that significantly improves the proposal of T. Keilmann et al. [Nat. Commun. 2, 361 (2011)], allowing as well for an exact realization of the two-body hard-core constraint, and for controllable effective interactions without the need of Feshbach resonances. We show that the combination of anyonic statistics and two-body hard-core constraint leads to a rich ground-state physics, including Mott insulators with attractive interactions, pair superfluids, dimer phases, and multicritical points. Moreover, the anyonic statistics results in a novel two-component superfluid of holon and doublon dimers, characterized by a large but finite compressibility and a multipeaked momentum distribution, which may be easily revealed experimentally.

DOI: 10.1103/PhysRevLett.115.053002

PACS numbers: 37.10.Jk, 67.85.-d, 05.30.Pr
\end{abstract}

Particles are classified as bosons or fermions depending on whether their wave function is symmetric or antisymmetric under exchange. Other types of quantum statistics are, however, possible in lower dimensions. Remarkably, 2D systems allow for the existence of anyons, i.e., particles with fractional statistics interpolating between bosons and fermions [1-3]. Anyons play a fundamental role in key areas of modern physics, as fractional quantum Hall effect [4-7] and topological quantum computing [8]. Fractional statistics is, however, not exclusive of 2D systems [9]. In particular, 1D anyons have attracted a large deal of interest [10-23], although the experimental realization of a 1D anyon gas is still lacking.

Ultracold atoms offer extraordinary possibilities for the analysis of interesting many-body systems [24]. In particular, several ideas have been proposed for the creation and manipulation of anyons in cold gases [25-29]. Particularly interesting is the recent proposal for the realization of the anyon Hubbard model (AHM) using Raman-assisted hopping in 1D optical lattices [30]. In this proposal, the anyonic statistics may be controlled at will, opening the possibility for the observation of statistically induced quantum phase transitions [30], asymmetric momentum distributions [21], and intriguing particle dynamics in the lattice $[20,22,23]$.

In this Letter, we first discuss a scheme for realizing the AHM that, although following Ref. [30], solves crucial drawbacks that would render the original proposal, in general, unfeasible. The scheme also allows for controllable effective interactions without the need of Feshbach resonances, and for an exact two-body hard-core constraint (2BHCC), contrary to the approximate realization of 2BHCC resulting from Zeno projection due to large three-body loss rates [31], where non-negligible losses are typically present. Neither the controllable interactions nor the inherent 2BHCC were considered in Ref. [30], which focused exclusively on statistically induced superfluid (SF) to Mott-insulator (MI) transitions. We show that the interplay of anyonic statistics, $2 \mathrm{BHCC}$, and controllable interactions, results in a far richer physics for the AHM, including pair superfluid (PSF), a dimer (D) phase, and an exotic partially paired (PP) phase. The latter constitutes, to the best of our knowledge, a novel two-component superfluid characterized by a large, but finite, compressibility and a multipeaked momentum distribution, which may be readily revealed in time-of-flight (TOF) experiments.

Anyon-Hubbard model.-We briefly describe our proposal for the realization of the AHM [32]. We consider atoms, bosons, or fermions, with states $|A\rangle$ and $|B\rangle$, in a deep tilted spin-independent 1D lattice with no direct hopping. For the specific case of ${ }^{87} \mathrm{Rb}$, the choice would be $|A\rangle \equiv\left|F=1, m_{F}=-1\right\rangle$ and $|B\rangle \equiv\left|F=2, m_{F}=-2\right\rangle$. Both states are coupled far from resonance by $D_{1}$ lasers $L_{1,4}$ (with linear polarization) and $L_{2,3}$ (with circular $\sigma_{-}$ polarization) [Fig. 1(a)]. Because of selection rules, $|B\rangle$ is just affected by lasers $L_{1,4}$. In contrast, both $L_{2,3}$ and $L_{1,4}$ couple with $|A\rangle$, but the coupling with $L_{1,4}$ can be made much smaller than that of $L_{2,3}$, and does not affect the main conclusions of this Letter [44]. We hence assume below that $|A\rangle$ is just affected by $L_{1,2}$. These lasers, with (a)

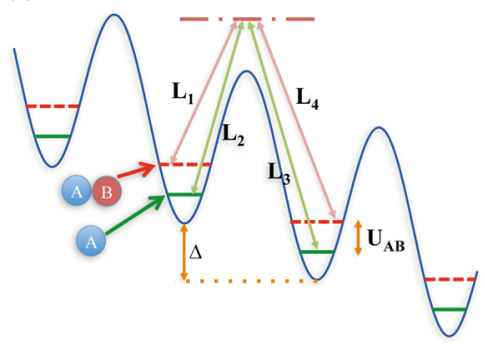

(b)

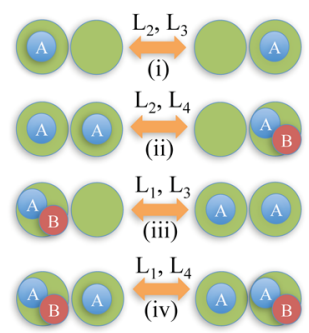

FIG. 1 (color online). (a) Raman scheme proposed for the realization of the AHM. (b) Raman-assisted hops (i)-(iv) discussed in the text. 
frequencies $\omega_{j=1, \ldots .4}$, induce four Raman-assisted hops [Fig. 1(b)]: (i) $(A, 0) \rightarrow(0, A)$, given by $L_{2,3}$, such that $\omega_{2}-\omega_{3}=-\Delta$ (with $\Delta$ the lattice tilting), (ii) $(A, A) \rightarrow$ $(0, A B)$, assisted by $L_{2,4}$ with $\omega_{2}-\omega_{4}=-\Delta+U_{A B}+U$, (iii) $(A B, 0) \rightarrow(A, A)$, given by $L_{1,3}$ with $\omega_{1}-\omega_{3}=$ $-\Delta-U_{A B}-U$, and (iv) $(A B, A) \rightarrow(A, A B)$, assisted by $L_{1,4}$, such that $\omega_{1}-\omega_{4}=-\Delta$. The notation $\left(\eta_{L}, \eta_{R}\right)$ denotes the state of neighboring sites $L$ and $R: \eta_{L, R}=0$ (vacuum), $A$ (single $A$ occupation), or $A B$ (an $A$ and a $B$ particle); $U_{A B}$ characterizes the $A B$ interaction. The frequency differences compensate the tilting, avoiding Bloch oscillations; moreover, the detuning $U$ acts as a controllable effective on-site interaction, as shown below.

We assume the width of the Raman resonances, $W$, is small enough such that each Raman process may be addressed independently. If the atoms are fermions, the only undesired process $(A, 0) \rightarrow(0, B)$ (where $B$ denotes a site with a single $B$ atom) may be avoided if $U_{A B} \gg W$. For bosons, $(A, A) \rightarrow(0, A A)$ and $(A B, A) \rightarrow(B, A A)$ (with $A A$ a site with two $A$ atoms) must be also avoided. This demands $U_{A A}-U_{A B}, U_{A A}, U_{A B} \gg W$, where $U_{A A}$ characterizes $A A$ interactions [32].

The laser $L_{j}$ is characterized by a Rabi frequency $\Omega_{j} e^{i \phi_{j}}$, with $\phi_{1}=-\phi$ and $\phi_{j \neq 1}=0$. We impose $\left(\left|\Omega_{1}\right|\left|\Omega_{4}\right| / 4\right)=$ $\left(\left|\Omega_{2}\right|\left|\Omega_{3}\right| / 3\right)=\left(\left|\Omega_{1}\right|\left|\Omega_{3}\right| / 2 \sqrt{3}\right)=\left(\left|\Omega_{2}\right|\left|\Omega_{4}\right| / 2 \sqrt{3}\right)=\Omega^{2}$ (for ${ }^{87} \mathrm{Rb}$, for other species Clebsch-Gordan coefficients may differ [32]). $\left|\Omega_{j}\right|$ is chosen such that they mimic bosonic enhancement, absent otherwise due to the distinguishability between $A$ and $B ; \epsilon=1(-1)$ for bosons (fermions). Under these conditions the system reduces to an effective single-component 1D Bose-Hubbard model [32], with on-site Fock states $\{|0\rangle,|1\rangle \equiv|A\rangle,|2\rangle \equiv|A B\rangle\}$, and an occupation-dependent Peierls phase [30,32]:

$H=-t \sum_{j}\left(b_{j}^{\dagger} e^{i \phi n_{j}} b_{j+1}+\right.$ H.c. $)+\frac{U}{2} \sum_{j} n_{j}\left(n_{j}-1\right)$,
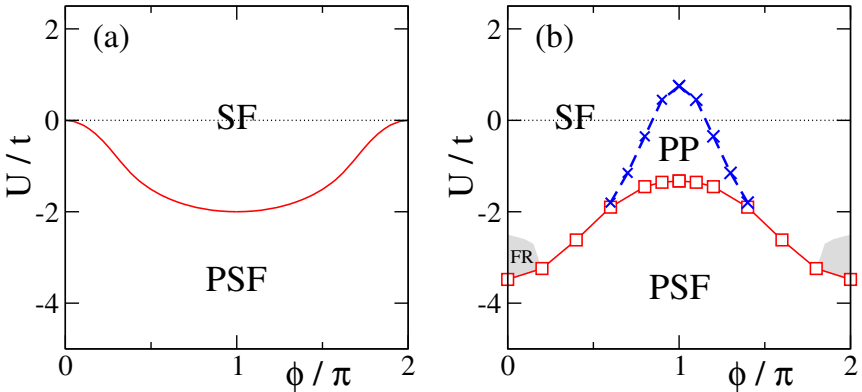

where $U$ is an effective coupling constant whose sign and magnitude may be tailored without the need of Feshbach resonances by properly choosing the laser frequencies, $t \simeq$ $J\left(\Omega^{2} / \delta\right) / 2 \Delta$ is the effective hopping rate [33], with $J$ the lattice hopping in the absence of tilting, and $\delta \gg U_{A B}, \Delta, \Omega$ the detuning from the single-photon transitions, $b_{j}^{\dagger}\left(b_{j}\right)$ are creation (annihilation) operators at site $j$, and $n_{j}=b_{j}^{\dagger} b_{j}$. Introducing $\alpha_{j} \equiv e^{i \phi \sum_{1 \leq l \leq j-1} n_{l}} b_{j}$, one obtains the AHM [30]:

$$
H=-t \sum_{j}\left(\alpha_{j}^{\dagger} \alpha_{j+1}+\text { H.c. }\right)+\frac{U}{2} \sum_{j} n_{j}\left(n_{j}-1\right),
$$

where $\alpha_{j}, \alpha_{j}^{\dagger}$ satisfy anyonic commutations [45]: $\alpha_{j} \alpha_{k}^{\dagger}-$ $e^{-i \phi \operatorname{sgn}(j-k)} \alpha_{k}^{\dagger} \alpha_{j}=\delta_{j k}$ and $\alpha_{j} \alpha_{k}-e^{-i \phi \operatorname{sgn}(j-k)} \alpha_{k} \alpha_{j}=0$.

None of the Raman-assisted hoppings leads to triple occupation, resulting in an effective $2 \mathrm{BHCC},\left(b_{j}^{\dagger}\right)^{3}=0$. This inherent constraint turns out to be crucial. It prevents collapse for a large $U / t<0$, leading to a rich physics of quantum phases (see Fig. 2) [46].

Note that although hops (i) and (iv) share the same energy difference $-\Delta$, they may be independently addressed, even if $\delta \gg U_{A B}, \Delta$. This point is crucial, solving the major drawback of Ref. [30] in which only one ground-state component was considered, and hence hops (i) and (iv) cannot be independently addressed unless $\delta \ll U_{A B}, \Delta$, which, for typical experimental parameters, would lead to large heating. An exception may be given by the use of clock transitions in alkaline-earth atoms [47]); however, the adiabatic elimination of the excited state demands $\Omega \ll \delta$, and hence $t \ll \delta \ll U_{A B}, \Delta$, would lead to very long experimental time scales [48].

Effective repulsion.-The dilute limit (lattice filling $\rho \ll 1$ ) is best understood from the analysis of the twoparticle problem [49], characterized by the scattering length (in units of the lattice spacing)
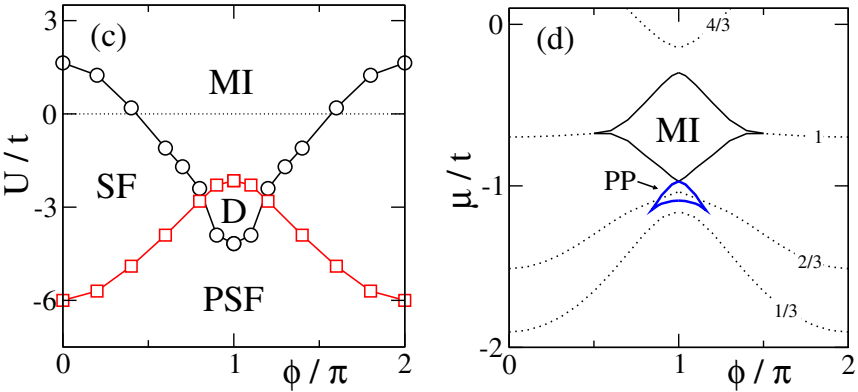

FIG. 2 (color online). Phase diagram of the AHM: (a) dilute limit $(\rho \rightarrow 0)$, (b) incommensurate intermediate fillings (here $\rho=1 / 2$ ), and (c) unit filling $(\rho=1)$. The PSF-SF transition lines [boxes or solid line in (a)], extracted from the crossing of two and one particle excitations coincides with the onset of parity order. At incommensurate fillings for small $\phi$, the system exhibits a first order transition from the SF to PSF phase through a forbidden region (FR) (dotted area). For $\phi / \pi \gtrsim 0.6$ we find the PP phase. For unit filling the Isingtype PSF-SF transition and the Kosterlitz-Thouless SF-MI transition lines cross around $\phi / \pi \approx 0.8$ in a multicritical point. For larger values of $\phi$ the system exhibits a dimerized gapped phase D. (d) Grand-canonical phase diagram and lines of constant density $\rho$ for $U=0$ as a function of $\mu$ and $\phi$. Solid lines denote the MI and PP phase; the rest is SF. For $\mu / t<-2(\mu / t>4$ ), the vacuum $\rho=0$ (fully occupied phase, $\rho=2$ ) is realized. 


$$
a=\frac{-(1+\cos \phi)}{4(1-\cos \phi)+2 U / t} .
$$

By comparison to a 1D gas of bosons with mass $m$ one extracts an effective interaction strength, $g=-2 /(a m)$ [49]. Thus, the AHM behaves as a repulsively interacting system for small $\rho$ and $U \rightarrow 0$, since even in that limit $g$ remains finite and positive for any $\phi>0$, approaching Tonks limit as $\phi \rightarrow \pi$. This is consistent with a weak coupling analysis [50], in which the Luttinger liquid parameter $K=\pi /\left[\phi^{2}+(U / 2 \rho t)\right]^{1 / 2}$, showing that $\phi$ has qualitatively the same effect as a repulsive $U>0$. In Fig. 2(a), we depict the phases for $\rho \rightarrow 0$ [51]: a SF phase, and a PSF of bound pairs at $U<0$. Because of the effective repulsion, the SF extends into the $U<0$ region for $\phi>0$.

Incommensurate fillings. - Bose models $(\phi=0)$ with $2 \mathrm{BHCC}$ have been recently studied in different scenarios $[31,52,53]$. A SF to MI transition results for $\rho=1$ and large-enough $U>0$; for $U<0$ a transition to a PSF occurs, which at incommensurate $\rho$ may become first order [54]. This FR occupies the shaded area in Fig. 2(b). At fixed $\rho$ the ground state is characterized by the formation of a macroscopically bound state with a phase separation of doublons and holes. The FR shrinks, however, fast with increasing $\phi$.

For $\phi \gtrsim 0.6 \pi$ a novel gapless phase, henceforth called PP phase, appears between the PSF and SF phases. As depicted in Fig. 3(a), a sharp kink in the $\rho(\mu)$ curve (at $\mu \simeq-t$ ) accompanies the onset of the PP phase. Whereas $\mathrm{SF}$ (PSF) is characterized by steps of 1 (2) particles in the $\rho(\mu)$ curve, the PP phase exhibits a complex sequence of steps of 2 and 1 particles. Even more relevant is the behavior of the central charge $c$, which we calculated from the conformal expression of the von Neumann entropy, $S_{v N, L}(l)=(c / 3) \ln [(L / \pi) \sin (\pi / L) l]+\gamma$, for a subsystem of length $l$ in a system of $L$ sites, with $\gamma$ a constant [55,56]. As shown in Fig. 3(b), contrary to the SF and PSF phases (with $c=1$ ), the PP phase is a two-component gapless phase characterized by $c=2$.
Nature of the PP phase.-The features of the PP phase may be understood from the following simplified picture. Because of the $2 \mathrm{BHCC}$, the hopping term in Eq. (1) is $J_{1} b_{j}^{\dagger} b_{j+1}+J_{2} b_{j}^{\dagger} n_{j} b_{j+1}$, with $J_{1} / t=1$ and $J_{2} / t=-1+$ $e^{i \phi}$. For $\phi \approx \pi$, correlated hopping dominates, $J_{2}=-2 J_{1}$, and doublons, or more precisely dimers, $|d\rangle_{j}=$ $\alpha|11\rangle_{j, j+1}+\beta|20\rangle_{j, j+1}$, gain a large binding energy $-\sqrt{2} J_{2}$. However, dimer hopping, $J_{1} / \sqrt{2}$, is reduced compared to single particles. For a certain range of small $U$ and $\rho$ it is energetically favorable to occupy both doublon dimers $\mid d>_{j}$ and atomic dimers, $|a\rangle_{j}=$ $\tilde{\alpha}|01\rangle_{j, j+1}+\tilde{\beta}|10\rangle_{j, j+1}$. Neglecting interactions between these two quasiparticles, reasonable for small doublon and atom densities, $\rho_{d}$ and $\rho_{a}$, with $\rho_{a}+\rho_{d} \ll 1$, one arrives at an effective model with two independent hardcore bosons, $H_{a d}=H_{a}+H_{d}$, with (for $U=0$ )

$$
\begin{gathered}
H_{a}=J_{1} \sum_{j}\left(a_{j}^{\dagger} a_{j+1}+\text { H.c. }\right)-J_{1} \sum_{j} a_{j}^{\dagger} a_{j}, \\
H_{d}=-\frac{J_{1}}{\sqrt{2}} e^{i \arg J_{2}} \sum_{j} d_{j}^{\dagger} d_{j+1}-\sqrt{2} J_{2} \sum_{j} d_{j}^{\dagger} d_{j},
\end{gathered}
$$

where $a_{j}\left(a_{j}^{\dagger}\right)$ and $d_{j}\left(d_{j}^{\dagger}\right)$ are annihilation (creation) operators of hardcore atom and doublon dimers on sites $j, j+1$, and $\rho_{a}+2 \rho_{d}=\rho$. In spite of being a gross oversimplification, $H_{a d}$ captures the qualitative features of the PP phase. For $U \approx 0$, at low $\rho$ the ground state only contains $a$ dimers, whereas for higher $\rho(\gtrsim 0.3$ for $U=0)$ both dimers are present.

The resulting $\rho(\mu)$ curve obtained from $H_{a d}$ (not shown) exhibits an irregular pattern of steps of 2 or 1 particles. The two-component phase extends for values around $U=0$, whereas for $U \gg J_{1}\left(U \ll-J_{1}\right)$ only $a(d)$ dimers are present [57].

The atom and doublon dimerizations, $N_{a}=\sum_{i}\left\langle b_{i}^{\dagger} b_{i+1}\right\rangle$ and $N_{d}=\sum_{i}\left\langle\left(b_{i}^{\dagger}\right)^{2}\left(b_{i+1}\right)^{2}\right\rangle$, approximately measure $\rho_{a}$ and $\rho_{d}$ [Fig. 3(c)]. Whereas the SF and PSF phases are Luttinger liquids of almost hardcore single particles or pairs
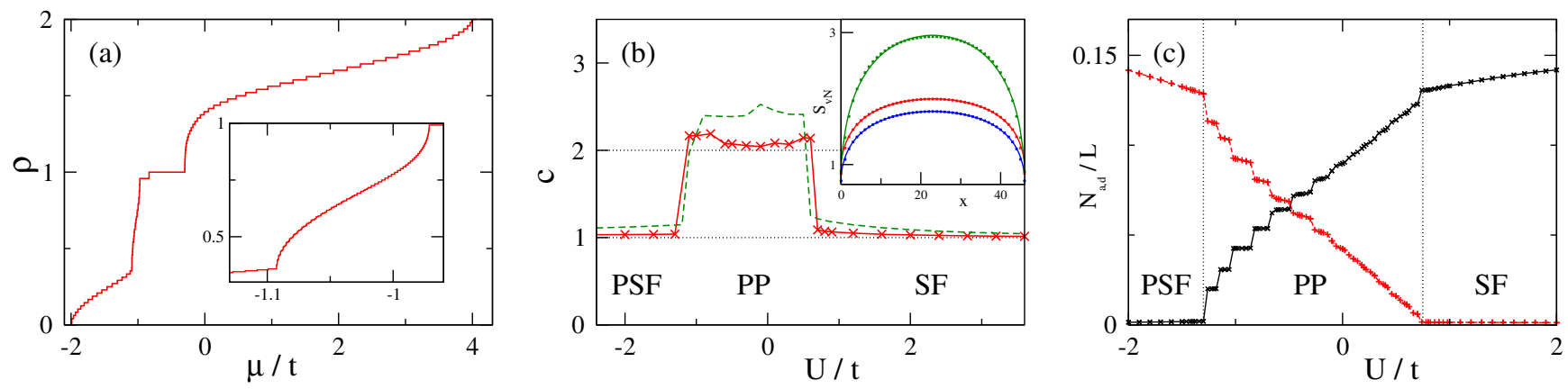

FIG. 3 (color online). Phase transitions for $\phi=\pi$. (a) Equation of state, density $\rho$ as function of chemical potential $\mu$ (calculated for $L=48$ sites, $U=0$ ). The inset shows a detailed view on the PP region for $L=240$ sites. (b) Estimation of the central charge for $L=24$ (dashed line) and $L=48$ (solid line) sites and periodic boundary conditions $(\rho=1 / 2)$. The inset shows fits to the blockentanglement entropy $S_{v N}$ for (from bottom to top) $U=2,-2,-1.0$. (c) Sum of local single- $\left(N_{a}\right)$ and two-particle correlations $\left(N_{d}\right)$ for $L=60$ sites and $\rho=1 / 2$, see text. 
(for $\phi=\pi$ ), in the PP phase both components coexist. Note the existence of many sharp jumps to states with approximately finite doublon density.

The AHM is characterized by a density- and $U$-dependent drift of the quasimomentum distribution [30,50], evident in Fig. 4 for the SF. Interestingly, whereas the SF presents an usual single-peaked distribution, the PP phase shows a multipeaked distribution. This is because the $a$ and $d$ hop with a different Peierls phase, and hence the overall $(a+d)$ momentum distribution presents multiple peaks. For PSF, single-particle correlations decay exponentially, and the peak structure blurs.

Unit filling.-We consider now the special case of $\rho=1$. Tuning $\phi$ may induce a SF to MI transition [30,50]. This is so even for $U<0$ [Fig. 2(c)] due to the effective on-site repulsion discussed above. This Kosterlitz-Thouless transition may be determined as the curve at which $K=2$ [58], which is consistent with the opening of the energy gap. As mentioned above, for $U<0$ an Ising-type transition occurs from SF to PSF. However, in the vicinity of $\phi \approx \pi$, the combination of anyonic statistics and $2 \mathrm{BHCC}$ results in a dimerized phase, $\mathrm{D}$. The latter is characterized by a finite dimer-order parameter $O_{D}=\left\langle T_{L / 2}-T_{L / 2+1}\right\rangle$ with $T_{i}=$ $b_{i}^{\dagger} b_{i+1}+$ H.c. The Ising transition line is determined by the crossing of single-and two-particle excitations, and by a sharp peak in the fidelity susceptibility [59]. Interestingly, the phase diagram presents a SF-MI-D-PSF multicritical point.

Probing the anyon-Hubbard model.-A discussion on experimental detection demands a grand-canonical analysis since local density arguments translate the dependence on the chemical potential, $\mu$, into a spatial dependence of the phases in the presence of an overall harmonic confinement. Crucially, the PP phase has a finite extension in both $\phi$ and $\mu$ [Fig. 2(d)]. Hubbard models with correlated hopping (both for fermions $[60,61]$ and bosons [62]) may present a similar (but insulating) phase with coexistent doublons and holons, which is, however, infinitely compressible; i.e., it is a single point as a function of $\mu$. As a result, these phases cannot be observed in trapped experiments, just leading to abrupt density jumps. On the contrary, the novel PP phase, having a finite $\mu$ extension, may be experimentally observable by properly setting the central $\mu$ in the PP region (adjusting the particle number).

Moreover, the multipeaked momentum distribution of the PP phase can be easily probed in TOF measurements. Figure 4(c) shows that TOF images of $A$ and the $B$ may be employed to reveal the PP phase [32].

Summary.-We propose a Raman scheme that realizes the AHM using cold atoms in 1D lattices, overcoming the main limitations of the proposal of Ref. [30]. This scheme results naturally in a $2 \mathrm{BHCC}$, and allows for tailoring effective on-site interactions without Feshbach resonances. The combination of anyonic character and 2BHCC leads to a rich ground-state physics, including Mott insulators for attractive interactions, a dimerized gapped phase, pair superfluids, and a novel two-component superfluid

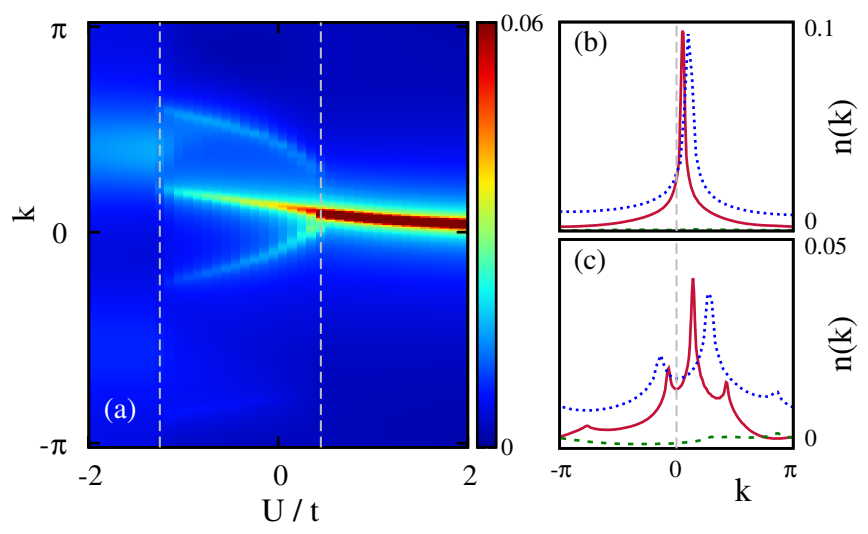

FIG. 4 (color online). (a) Quasimomentum distribution $n(k)$ for $\rho=1 / 2$ as a function of $U / t$ for $\phi=0.9 \pi$ ( $L=60$ sites). Dashed lines denote phase transitions between PSF, PP, and SF phases. (b) and (c) show the momentum distribution for $U=2 t$ and $U=0$ for the AHM (solid line) and the components $A$ (dashed line) and $B$ (dotted line) separately (for bosonic atoms). The latter are obtained by direct simulation of the two-component model with Raman-induced hopping (see the Supplemental Material [32] for a discussion of the fermionic case).

(partially paired phase) characterized by a large compressibility and a peculiar multipeaked momentum distribution that may be easily probed experimentally. Finally, we would like to mention that the Raman scheme may be extended to 2D lattices. Although for 2D lattices the bosonanyon mapping is lost, a 2D setup would open the exciting possibility of inducing a density-dependent magnetic field, contrary to the static field created in recent experiments [33,63-65]. This possibility will be investigated in a forthcoming work.

We thank A. Eckardt, C. Klempt, D. Poletti, M. Roncaglia, E. Tiemann, and T. Vekua for discussions. We acknowledge support by the Cluster QUEST and the DFG Research Training Group 1729. Simulations were carried out on the cluster of the Leibniz Universität Hannover.

[1] J. M. Leinaas and J. Myrheim, Nuovo Cimento B 37, 1 (1977).

[2] F. Wilczek, Phys. Rev. Lett. 49, 957 (1982).

[3] G. S. Canright and S. M. Girvin, Science 247, 1197 (1990).

[4] R. B. Laughlin, Phys. Rev. Lett. 50, 1395 (1983).

[5] B. I. Halperin, Phys. Rev. Lett. 52, 1583 (1984).

[6] F. E. Camino, W. Zhou, and V. J. Goldman, Phys. Rev. B 72, 075342 (2005).

[7] E.-A. Kim, M. Lawler, S. Vishveshwara, and E. Fradkin, Phys. Rev. Lett. 95, 176402 (2005).

[8] C. Nayak, S. H. Simon, A. Stern, M. Freedman, and S. Das Sarms, Rev. Mod. Phys. 80, 1083 (2008).

[9] F. D. M. Haldane, Phys. Rev. Lett. 67, 937 (1991).

[10] F. D. M. Haldane, Phys. Rev. Lett. 66, 1529 (1991).

[11] Z. N. C. Ha, Phys. Rev. Lett. 73, 1574 (1994).

[12] M. V. N. Murthy and R. Shankar, Phys. Rev. Lett. 73, 3331 (1994).

[13] Y.-S. Wu and Y. Yu, Phys. Rev. Lett. 75, 890 (1995). 
[14] J.-X. Zhu and Z. D. Wang, Phys. Rev. A 53, 600 (1996).

[15] L. Amico, A. Osterloh, and U. Eckern, Phys. Rev. B 58, R1703 (1998).

[16] A. Kundu, Phys. Rev. Lett. 83, 1275 (1999).

[17] M. T. Batchelor, X.-W. Guan, and N. Oelkers, Phys. Rev. Lett. 96, 210402 (2006).

[18] M. D. Girardeau, Phys. Rev. Lett. 97, 100402 (2006).

[19] P. Calabrese and M. Mintchev, Phys. Rev. B 75, 233104 (2007).

[20] A. del Campo, Phys. Rev. A 78, 045602 (2008).

[21] Y.Hao, Y. Zhang, and S. Chen, Phys. Rev. A 79, 043633 (2009).

[22] Y. Hao and S. Chen, Phys. Rev. A 86, 043631 (2012).

[23] L. Wang, L. Wang, and Y. Zhang, Phys. Rev. A 90, 063618 (2014).

[24] I. Bloch, J. Dalibard, and W. Zwerger, Rev. Mod. Phys. 80, 885 (2008).

[25] B. Paredes, P. Fedichev, J. I. Cirac, and P. Zoller, Phys. Rev. Lett. 87, 010402 (2001).

[26] L.-M. Duan, E. Demler, and M. D. Lukin, Phys. Rev. Lett. 91, 090402 (2003).

[27] A. Micheli, G. K. Brennen, and P. Zoller, Nat. Phys. 2, 341 (2006).

[28] M. Aguado, G. K. Brennen, F. Verstraete, and J. I. Cirac, Phys. Rev. Lett. 101, 260501 (2008).

[29] L. Jiang, G. K. Brennen, A. V. Gorshkov, K. Hammerer, M. Hafezi, E. Demler, M. D. Lukin, and P. Zoller, Nat. Phys. 4, 482 (2008).

[30] T. Keilmann, S. Lanzmich, I. McCulloch, and M. Roncaglia, Nat. Commun. 2, 361 (2011).

[31] A. J. Daley, J. M. Taylor, S. Diehl, M. Baranov, and P. Zoller, Phys. Rev. Lett. 102, 040402 (2009).

[32] See Supplemental Material at http://link.aps.org/ supplemental/10.1103/PhysRevLett.115.053002, which includes Refs. [33-43], for details and concrete examples.

[33] H. Miyake, Ph.D. thesis, Massachusetts Instittute of Technology, 2013.

[34] D. A. Steck, Rubidium 87D Line Data, http://steck.us/ alkalidata (revision 2.1.4, 12/23/2010).

[35] D. A. Steck, Rubidium 85D Line Data, http://steck.us/ alkalidata (revision 2.1.6, 9/20/2013).

[36] D. A. Steck, Sodium D Line Data, http://steck.us/alkalidata (revision 2.1.4, 12/23/2010).

[37] D. A. Steck, Cesium D Line Data, http://steck.us/alkalidata (revision 2.1.4, 12/23/2010).

[38] T. G. Tiecke, Ph.D. thesis, University of Amsterdam, 2009.

[39] J. S. Krauser, J. Heinze, N. Fläschner, S. Götze, C. Becker, and K. Sengstock, Nat. Phys. 8, 813 (2012).

[40] Y. Kawaguchi and M. Ueda, Phys. Rep. 520, 253 (2012).

[41] J. F. Sherson, C. Weitenberg, M. Endres, M. Cheneau, I. Bloch, and S. Kuhr, Nature (London) 467, 68 (2010).

[42] E. Tiemann (private communication).

[43] J. Kronjäger, Ph.D. thesis, Universität Hamburg, 2007.

[44] The spurious coupling of $|B\rangle$ with $L_{2,3}$ leads to additional small terms in the final Hamiltonian, which, as discussed in the Supplemental Material [32], do not affect the main results of this Letter.

[45] E. Fradkin, Field Theories of Condensed Matter Systems (Addison-Wesley, Redwood City, CA, 1991).
[46] For our analysis of the ground-state physics, we performed large-scale density matrix renormalization group calculations [see, e.g., U. Schollwöck, Ann. Phys. (Amsterdam) 326, 96 (2011)] with open and periodic boundary conditions. Keeping up to 800 matrix states we simulate systems with up to $\boldsymbol{L}=240$ sites for open boundary conditions and $\boldsymbol{L}=48$ sites for periodic boundary conditions.

[47] See, e.g., M. A. Cazalilla and A. M. Rey, Rep. Prog. Phys. 77, 124401 (2014), and references therein.

[48] Typical experimental values are $U_{A B}$ and $\Delta$ of the order of $100 \mathrm{~Hz}$ to few $\mathrm{kHz}, \Omega$ of tens of kHz, $\delta$ of few GHz, and hence $t$ of the order of few $\mathrm{kHz}$. Demanding $\delta \ll U_{A B}, \Delta$, hence requires $\Omega \ll \delta \ll$ few $\mathrm{kHz}$, and hence $t$ well below $1 \mathrm{~Hz}$.

[49] A. K. Kolezhuk, F. Heidrich-Meisner, S. Greschner, and T. Vekua, Phys. Rev. B 85, 064420 (2012).

[50] S. Greschner, G. Sun, D. Poletti, and L. Santos, Phys. Rev. Lett. 113, 215303 (2014).

[51] While Eq. (3) correctly describes the effective repulsive behavior for positive and $U<0$, this simplified two-particle scattering picture does not capture the correct SF-PSF transition line for $\phi \rightarrow \pi$ and $U<0$, as shown in Fig. 2(a).

[52] L. Bonnes and S. Wessel, Phys. Rev. Lett. 106, 185302 (2011).

[53] S. Greschner, L. Santos, and T. Vekua, Phys. Rev. A 87, 033609 (2013).

[54] This may be understood from known results of spin-1 models [see T. Tonegawa, K. Okunishi, T. Sakai, and M. Kaburagi, Prog. Theor. Phys. Suppl. 159, 77 (2005)], where for negative single-ion anisotropies (i.e., $\boldsymbol{U}<0$ in the boson language) the transition between $\boldsymbol{X} \boldsymbol{Y}$ and $\boldsymbol{X Y} 2$ phases (i.e., SF and PSF phase) is first order passing a region of macroscopic jumps in magnetization as a function of the magnetic field (i.e., in $\rho$ as a function of chemical potential, $\boldsymbol{\mu}$ ) for small magnetizations (i.e., close to unit filling, $\boldsymbol{\rho}=1$ ). For other fillings the phase transition may become second order.

[55] G. Vidal, J. I. Latorre, E. Rico, and A. Kitaev, Phys. Rev. Lett. 90, 227902 (2003).

[56] P. Calabrese and J. Cardy, J. Stat. Mech. (2004) P06002.

[57] The effective model $H_{a d}$ suggests the SF-PP transition to be of a commensurate-incommensurate type. This is consistent with the numerically observed cusp singularity in the $\rho(\mu)$ curve, as shown in Fig. 3(a).

[58] Th. Giamarchi, Quantum Physics in One Dimension, (Oxford University Press, New York, 2004).

[59] S. Greschner, A. K. Kolezhuk, and T. Vekua, Phys. Rev. B 88, 195101 (2013).

[60] A. A. Aligia, K. Hallberg, C. D. Batista, and G. Ortiz, Phys. Rev. B 61, 7883 (2000).

[61] M. Di Liberto, C. E. Creffield, G. I. Japaridze, and C. Morais Smith, Phys. Rev. A 89, 013624 (2014).

[62] Á. Rapp, X. Deng, and L. Santos, Phys. Rev. Lett. 109, 203005 (2012).

[63] M. Aidelsburger, M. Atala, M. Lohse, J. T. Barreiro, B. Paredes, and I. Bloch, Phys. Rev. Lett. 111, 185301 (2013).

[64] H. Miyake, G. A. Siviloglou, C. J. Kennedy, W. C. Burton, and W. Ketterle, Phys. Rev. Lett. 111, 185302 (2013).

[65] M. Atala, M. Aidelsburger, M. Lohse, J. T. Barreiro, B. Paredes, and I. Bloch, Nat. Phys. 10, 588 (2014). 